\title{
Uncovering Interface Structure by Column Shape Analysis in ADF STEM Images
}

\author{
A.Y. Borisevich, ${ }^{*}$ O. Ovchinnikov, ${ }^{* *}$ H.J. Chang, ${ }^{*}$ M.P. Oxley, ${ }^{*}$ P. Yu, ${ }^{* * *}$ R. Ramesh, ${ }^{* * *}$ S.J. \\ Pennycook, ${ }^{*}$ S.V. Kalinin ${ }^{*}$ \\ * Oak Ridge National Laboratory, Oak Ridge, TN 37831 \\ ${ }^{* *}$ University of Tennessee, Knoxville, TN 37996 \\ *** Department of Materials Science and Engineering and Department of Physics, University of \\ California, Berkeley, CA 94720
}

The multifaceted magnetic, electrical, and structural functionalities of perovskite $\mathrm{ABO}_{3}$ materials are underpinned by the subtle distortions of the crystallographic lattice from cubic prototype. These distortions include relative displacements of the cations from the centers of the $\mathrm{BO}_{6}$ oxygen octahedra, deformations of oxygen octahedra, and collective tilts of octahedral network. Further interest in this behavior has emerged from the fabrication of novel heterointerfaces that allow new functionalities that do not exist in the bulk, including interface superconductivity, improper ferroelectricity, and magnetoelectric coupling phenomena. Furthermore, at homophase interfaces such as ferroelastic domain walls, symmetry changes alone often give rise to new properties.

With the advent of the aberration correction, it became possible to map structural distortions on a unit cell scale, from polarization $[1,2]$ to octahedral tilts $[3,4]$. However, the analysis based on geometric calculation of tilts requires simultaneous detection of cation and anion positions, limiting the usable sample thicknesses to values under $10 \mathrm{~nm}$ which are difficult to achieve routinely. Additionally, very thin areas can be prone to beam damage and elastic relaxation, and/or exhibit significant surface and size effects, complicating interpretation of the results.

Here we introduce a new paradigm for order parameter mapping in complex oxides based on a direct shape analysis of Z-contrast images of the heavy atomic columns, where the details of column shape contain the information on the positions of unresolved light atom columns. This method can be used to map, unit cell by unit cell, octahedral tilt patterns in the vicinity of hetero- and homointerfaces. Our approach is to use a grid of small patches of the image centered on atomic columns as a "spectrum image" for PCA-based analysis. The output eigenvectors (eigenshapes) are elementary column images that seem to reflect either average shape ( $1^{\text {st }}$ component) or a particular shape distortion (subsequent components). The spatial distribution and spatial correlations of the distortion maps reflect the underlying material structure.

Rhombohedrally distorted perovskites such as $\mathrm{BiFeO}_{3}(\mathrm{BFO})$ provide great demonstration materials for the technique. In BFO, the octahedral network viewed along some of the pseudocubic $<110>$ directions shows a "checkerboard" pattern of the tilts of the octahedra (Fig. 1(a)). While so far imaging of the tilted octahedra relied on the detection of equatorial oxygen atoms (located between Fe columns), our PCA-based technique can detect the distortions in the shape of the Bi columns caused by their incomplete overlap with axial oxygen atoms. Fig. 1(b) shows a portion of a high angle annular dark field (HAADF) STEM image of a $5 \mathrm{~nm}$ BFO thin film grown on a $\mathrm{SrTiO}_{3} / \mathrm{SrRuO}_{3}(\mathrm{SRO})$ substrate. The equatorial oxygen atoms are not visible in this image. Next we conduct shape analysis on the Bi sublattice (Fig.1(c)-(h)). The first eigenshape (Fig. 1(c)) clearly 
corresponds to the average shape, showing contrast between two materials due to different overall intensity of the columns (Fig. 1(f)). The next component (Fig. 1(d)) reflects an overall distortion of the columns and the corresponding map (Fig. 1(g)) does not have a clear tie to the crystal structure; it could be related to a small bend in the sample that increases towards the thinnest area in the upper right. The third component (Fig. 1(e)) describes diagonal distortion associated with the overlapping oxygen columns, and the corresponding map (Fig. 1(h)) shows clear checkerboard contrast in the $\mathrm{BFO}$ but not in SRO. The same approach can be used to analyze images of domain walls in BFO (Fig. 2). The use of the method is validated by image simulations [5]. This work is a part of a larger effort to learn to extract all the information from high resolution STEM images using quantitative image analysis [6].

In summary, column shape analysis is a quick and robust method of detecting evolution of structural patterns at the interfaces in oxides, enabling us to extract high-level structural information from virtually every atomic resolution image. Discussions on quantitative determination of lattice distortions from the PCA data and the correlation to the geometric measurements of the octahedral tilts on the same images will also be presented.

\section{References}

[1] C.L. Jia et al. , S. Choudhury et al., Nature Mat. 6 (2007) 64.

[2] C.L. Jia et al., Nature Mat. 7 (2008) 57

[3] C.L. Jia et al., Phys. Rev. B 79 (2009) 08140

[4] A. Borisevich et al., arXiv:1002.2989v1 [cond-mat.mtrl-sci]

[5] M.P. Oxley et al., these proceedings

[6] O. Ovchinnikov et al., these proceedings

[7] This research is sponsored by the Division of Materials Sciences and Engineering, Office of BES of the U.S. DOE, and by appointment (H.J.C.) to the ORNL Postdoctoral Research Program administered jointly by ORNL and ORISE.

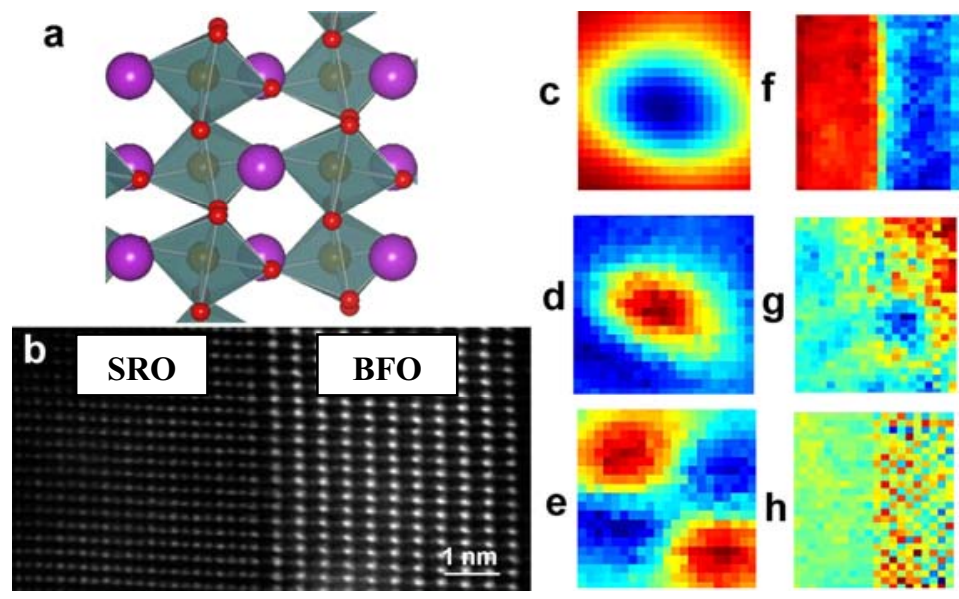

Fig.1. Column shape analysis: (a) structure of BFO in one of the $<110>$ pseudocubic projections; (b) HAADF image of the SRO-BFO interface used for analysis, (c)-(e) first 3 eigenshapes and $(\mathrm{f})-(\mathrm{h})$ the corresponding maps.
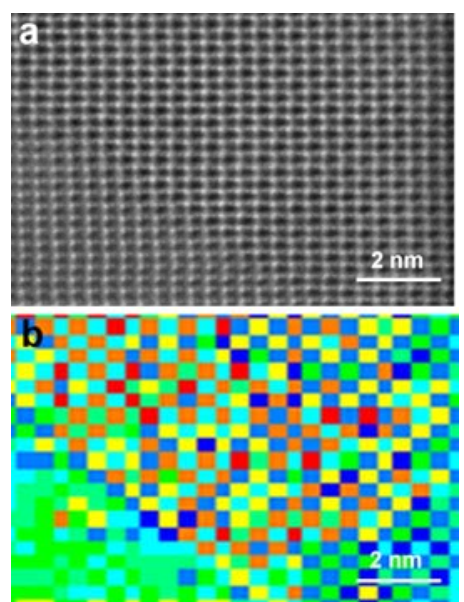

Fig.2. (a) HAADF STEM image of a domain wall in a BFO thin film; (b) the map for the third eigenshape showing checkerboard order on one side of the wall and not the other, reflecting different orientations of the rhombohedral lattice. 